\title{
Jahres-Inhalt 1989, Vol. 12, 1989
}

Hiddemann, W. (Münster) et al.

Altersbezogener randomisierter Vergleich von sequen-

tiell appliziertem hochdosiertem versus intermediär dosiertem Cytosin-Arabinosid in Kombination mit Mitoxantron (S-HAM) in der Therapie rezidivierter und refraktärer akuter myeloischer Leukämien: Studienkonzept und vorläufige Ergebnisse

Linkesch, W. (Wien/Österreich) et al.

Amsacrine, Cytarabin und Thioguanin (AAT) versus

Daunorubicin, Cytarabin, Thioguanin (DAT) bei Erwachsenen mit unbehandelter akuter nicht-lymphatischer Leukämie (ANLL): Ergebnisse aus Österreich und der Bundesrepublik Deutschland Informationen für die Klinik Ganser, A.; Ottmann, G.; Hoelzer, A. (Frankfurt), Schulz, G. (Marburg)

Therapie von Patienten mit myelodysplatischen Syndro-men mit Granulozyten-MakrophagenKoloniestimulie-rendem Faktor (rhGM-CSF) und Cytosin-Arabinosid . .

Gattringer, C; Thaler, J.; Orach, J.;

Huber, H. (Innsbruck/Österreich),

Micksche, M. (Traiskirchen/Österreich)

GM-CSF Behandlung in der Aplasie nach zytostatischen

Therapien

Steinke, B. (Tubingen) et al.

Sequentiell alternierende Chemotherapie bei hochmalignen Non-Hodgkin-Lymphomen mit VIM-Bleo und CHOP. Erste Ergebnisse

Gerhartz, H. H. (München) et al.

Risiko-adaptierte Therapie hochmaligner Non-Hodgkin-

Lymphome mit COP-LBAM/IMVP-16: Eine prospektive multizentrische Studie

Preis, P. (Wien/Österreich) et al.

VMCP-Chemotherapie mit oder ohne Interferon-alpha-2 bei neudiagnostizierten Patienten mit multiplem Myelom

Sonderbände . .

Hartenstein, R.C.; Wendt, T.G.; Kastenbauer, E.R.

(München), Trott, K.R. (London/GB)

Simultane Chemo-Radiotherapie mit 5-Fluorouracil/Folinsäure/Cisplatin und hyperfraktionierter Bestrahlung bei fortgeschrittenen HNO-Karzinomen 
Buchbesprechungen

Voigtmann, R. (Bochum)

Knochenmarksnekrose bei einer Patientin mit metastasierendem Mammakarzinom unter Chemotherapie mit Chlorambucil, Methotrexat und Prednison 36

Kurzmitteilungen 4 Karstens, J.H.; Schnabel, B.; Ammon, J. (Aachen)

Behandlung von metastasenbedingtem Knochenschmerz:

erste Ergebnisse einer Studie mit Einzelfraktions-

(4 Gy) Strahlentherapie

41

Sommerkamp, H. (Freiburg)

Eingeschränkte Therapie beim Prostatakarzinom 44

8

9 Abstrakts vom Symposium der Arbeitsgemeinschaft für internistische Onkologie (AIO) - Neue Perspektiven in der Chemotherapie maligner Neoplasien, Hamburg,

Februar1989 52

Kabisch, H.; Niggemann, B.; Winkler, K. (Hamburg) 12 Extreme Hyperphosphatämie mit Hypokalzämie im Rah-

men eines Zellyse-Syndromes bei einem Kind mit T-ALL 64

Nagel, G. A.; Ammon, A.; Holtkamp, W.; Meyer, D. (Göttingen)

Klinische Modellvorstellung der Evolution maligner 16 Tumoren: Implikationen für die

Hormontherapie des

Mammakarzinoms 69

Aulbert, E. (Berlin), Jakob, M.; Kurschel, E. (Essen) Die Anämie bei malignen

Tumorerkrankungen - V. Die -*" Beziehung zwischen der tumorbedingten Hypotrans-

ferrinämie und dem Grad der Anämie $\quad 81$

Buchbesprechungen 90

22 Winkler, K.; Torggler, S.; Beron, G.; Steinhoff, A.

(Hamburg/Eppendorf), Bode, U. (Bonn), Gerein, V.

(Frankfurt), Jürgens, H. (Düsseldorf), Kusnierz-Glaz, C.

(Münster), Kotz, R.; Salzer-Kuntschik, M.

27

(Wien/Osterreich), Schmoll, H. J. (Hannover)

$2 \cap$ Behandlungsergebnisse bei primär disseminiertem Osteo-

sarkom. Verlaufsanalyse von Patienten aus den coopera-

tivenOsteosarkom-StudienCOSS-80undCOSS-82 ... 92

Obrist, R. (Basel/Schweiz), Honegger, H. P.

(Zürich/Schweiz), Aapro, M. (Genf/Schweiz), Bürki, K. 3Q (Bern/Schweiz), Cavalli, F.

(Bellinzona/Schweiz)

ABLETOP, ein gut toleriertes, effektives Chemothera-35 pie-Regime für den

rezidivierendenMorbusHodgkin. . . 99

310

Jahres-Inhalt 1989

Kurzmitteilung

Runge, H.-M.; Meerpohl, H. G.; Pfleiderer, A. 
(Freiburg i.Br.)

Mitoxantron in der Therapie des fortgeschrittenen Ade-

nokarzinoms des Endometriums. Ein Phase-II-Versuch. . 102

Informationen für die Klinik 104

Editorial 108

Völkers, B.; Ganser, A.; Greher, J.; Hoelzer, D. (Frankfurt), Carlo Stella, C. (Pavia/Italien)

Untersuchungen über die Wirkung von Tumornekrose-faktor auf humane hämatopoetische Vorläuferzellen . . . 109 Burdach, S.; Wahn, V.; Göbel, U. (Düsseldorf) , Levitt, L. (Stanford, Calif./USA)

Lymphokin-vermittelte Suppression der Erythropoese durch normale T-Lymphozyten und bei Retrovirus-asso-

ziierter lymphoproliferativer Erkrankung 116

Hamprecht, K. G.; Vötsch, W.; Anderer, F. A.

(Tubingen)

Ein dialysabler saurer Faktor aus Human-Leukozyten-

Extrakten aktiviert die Humanmonozyten- und Natür-

liche-Killer-Zell-vermittelteTumorzell-Lyse 120

Pfreundschuh, M.; Genth, B.; Steinmetz, H. T.; Schaadt,M.; Diehl, V. (Köln), Kirchner,H.

(Hannover), Scheurich, P. (Göttingen), Lindemann, A. (Mainz) Untersuchungen zum

Resistenzmechanismus gegen Tu-

mor-Nekrose-Faktor 128

Kallinowski, F.; Moehle, R.; Schaefer, C.; Vaupel, P.

(Mainz)

Wirkungen von Tumor-Nekrose-Faktor- $\alpha$ auf die Tumor-

perfusion und die Effizienz einer Hyperthermiebe-

handlung 131

Bartsch, H. H.; Pfizenmaier, K.; Schroder, M.;

Nagel, G. A. (Göttingen)

Systemische versus lokale Therapie mit rekombinantem

Tumor-Nekrose-Faktor-alpha (r-TNF-alpha) bei Patien-

ten mit fortgeschrittenen Tumoren 136

Informationen für die Klinik 142

Klapdor, R.; Franke, N.; Bahlo, M. (Hamburg) Kombinierte Therapie von Xenotransplantaten humaner Pankreaskarzinome mit rTNF-alpha und Mitomycin-C . 143

AIO-Mitteilungen 148

Buchbesprechungen 152

Queißer, W.; Heim, M. E. (Mannheim)

Kombinierte Strahlen- und Chemotherapie beim Magen-

karzinom. Eine Übersicht 156

Heim, M. E.; Schuster, D.; Flechtner, H.; Worst, P.; Queißer, W. (Mannheim)

Sequentielle Therapie mit hochdosiertem Methotrexat,

5-Fluorouracil und Folinsäure verbessert die Ansprech-

raten beim fortgeschrittenen kolorektalen Karzinom

nicht 161

Jäger, W.; Adam, R. (Erlangen) 
Vergleich der Serumkonzentrationen des CA-72.4 mit dem CA-125 während des klinischen Verlaufs von Ovarialkarzinom-Patientinnen 164

Fischer, H.; Zeller,W.J. (Heidelberg); Schwechheimer, K. (Freiburg), Flutter, K.-J.; Wowra, B.;

Kunze, St. (Heidelberg), Sturm, V. (Köln)

Etablierung und Charakterisierung von menschlichen

Glioblastomlinien in vitro und ihre Xenografts in der

Nacktmaus 169

Gabius, H.-J. (Göttingen)

Endogene Lektine in Tumoren und ihre mögliche Bedeu-

tung für Diagnose und Therapie von Krebserkrankungen . 175

Informationen für die Klinik 182

Rink, B.; Hiepe, F.; Apostoloff, E. (Berlin)

Zirkulierende Immunkomplexe bei Patienten mit Mund-

schleimhautkarzinomen

183

Kurzmitteilungen

Karstens, J. H.; Hermes, H.; Schnarkowski, P.;

Ammon, J. (Aachen)

Beschleunigte fraktionierte Bestrahlung bei gleichzeitiger

Cisplatin-Infusion bei fortgeschrittenem Kopf- und Hals-

krebs 187

Laskawi, R.; Brauneis, J. (Göttingen); Schroder, M. (Münster); Göhde, W. (Kassel)

Die prognostische Bedeutung der Impulszytophotometrie

(ICP) bei Malignomen des Kopf-Hals-Bereiches 190

Hemmer, J.; Kreidler, J.; Haase, S. (Ulm)

DNS-Ploidie bei Basaliomen des Kopfes 192

AIO-Mitteilungen 194

Editorial 200

Queißer, W.; Flechtner, H.; Heim, M.E. (Mannheim); Kabelitz, K. (Homburg/Saar); Maßner, B.

(Frankenthal); Edler, L. (Heidelberg)

Dosiseskalation und -modifikation von 4-Epidoxorubicin

in der Kombinationschemotherapie (FEM II) des fortge

schrittenen Magenkarzinoms 202

Jahres-Inhalt 1989

311

Günczler, P.; Ogris, E.; Maca, S.; Danmayr, E.

(Wien/Österreich)

Tumormarker bei Mammakarzinom: Zur diagnostischen

Wertigkeit von Serumbestimmungen bei klinischer Tumor-

freiheit (NED) und manifester Erkrankung 209

Meden, H.; Rath, W.; Teichmann, A.; Kuhn, W.

(Göttingen)

Serumspiegel des Tumormarkers CA125 vor der Second-

look-Laparotomie beim primären epithelialen Ovarial-

karzinom in Relation zu intraoperativen Befunden .... 217 
Buchbesprechungen 221

Informationen für die Klinik 224

Hausmaninger, H. (Salzburg/Österreich); Lehnert, M.; Samonigg, H. (Graz/Österreich); Steger,

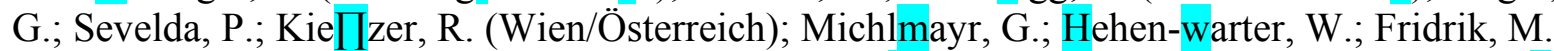
(Linz/Österreich); Schiller, L. (Vöcklabruck/Österreich); Manfreda, D. (Klagenfurt/ Österreich); Haidinger, R. (Steyr/Österreich); Neiss, A. (Innsbruck/Österreich)

Vindesin-Epirubicin (VE) versus Vindesin-Mitoxantron

(VM) beim metastasierenden Mammakarzinom 225

Hallek, M.; Haen, E.; Emmerich, B. (München);

Lévi, F. (Villejuif/Frankreich)

Bedeutung der Chronopharmakologie für die Onkologie

230

Manegold, C; Bülzebruck, H.; Drings, P.; Vogt-Moykopf, I. (Heidelberg)

Prognostische Faktoren beim kleinzelligen Bronchial-

karzinom 240

AIO-Mitteilungen 246

Garbe, C; Orfanos, C.E. (Berlin)

Epidemiologie des malignen Melanoms in der Bundes-

republik Deutschland im internationalen Vergleich .... 253

Breitbart, E.W.; Mohsenían, F; Roser, M.; Schiers, C;

Wíebecke, G.-E; Reimitz, P.E.;

Höhne, K.H. (Hamburg)

Untersuchungen zur klinischen Erkennbarkeit der Früh-

formen des malignen Melanoms 264

Landthaler, M.; Braun-Falco, O. (München)

Zur Therapie des malignen Melanoms im Stadium I.

Offene Fragen und Empfehlungen 269

Lehmann, J.M.; Holzmann, B.; Rothbächer, U.;

Riethmüller, G.; Johnson, J.P. (München)

Schrittweise Expression von Melanom-assoziierten Anti-

genen während der Tumorprogression 273

Koeppler, H.; Pflueger, K.H.; Seitz, R-;

Havemann, K. (Marburg)

Hochdosierte Chemotherapie mit autologer Knochen-

marktransplantation beim metastasierten malignen

Melanom 277

Voigt, H.; Aigner, K.R.; Link, K.H.; Walther, H.; Mutter, H. (Ulm)

Systemisch applizierte regionale Tumortherapie. Regio-

nale Halbkörperchemotherapie des metastasierenden

malignen Melanoms - eine experimentelle Therapiekon-

zeption 280

Roser, M.; Frenzer, S.; Schmidt-Preuss, U,; Reimers, U.;

Weíchenthal, M.; Breitbart, E. W.;

Rüdíger, H. W. (Hamburg)

Chromosomale Instabilität bei Patienten mit malignem

Melanom der Haut 286 
Weyer, U.; Peter sen, I.; Ehrke, C; Carstensen, A.;

Nüßgen, A.; Russ, C; Göttsch, B.; Kowalzíck, L.;

Arndt, R.; Breitbart, E. W. (Hamburg)

Immunomodulation durch Kryochirurgie beim malignen

Melanom 291

Abstrakts zum Symposium der AIO

«lnterferone - Biologische Grundlagen und klinische An-

wendung», 26727. Januar 1990 in Hamburg 297

AIO-Mitteilungen 305

Autorenverzeichnis $1989 \quad 308$

Jahres-Inhalt 1989309

312

Jahres-Inhalt 1989

Supplement 1

Editorial 3

Schalk, K. P.; Rütteńans, H.; Kaltwasser, J. P.; Said l-Hadj; Staffenberger, L. (Frankfurt)

Tumorerkennung im Serum durch NMR-Spektrometrie. Eine kritische Überprüfung des

sogenannten Fossel-Tests 5

Gussetis, E. S.; Ebener, U.; Wehner, S.; Kornhuber, B.

(Frankfurt)

Immunologische Identifizierung von durch herkömmliche

zytohistologische Untersuchungen an Knochenmarkspro-

ben nicht erkennbare Neuroblastomzellen 9

Bares, R.; Faß, J.; Weiller, G.; Wolter, S.; Kleinhans, E.; Truong, S.; Bull, U.; Schumpelick, V.

(Aachen) Klinische Bedeutung der Immunszintigraphie für Diagnose und Therapie

gastrointestinaler Malignome .... 13

Halting, Th.; Steinbächer, M.; Kretzschmar, U.;

Georgi, P.; Schlag, P. (Heidelberg)

Verbessert die Immunszintigraphie die Frühdiagnostik

des metastasierten oder rezidivierten Karzinoms? 20

Baum, R. P.; LoreПz, M.; Hertel, A.;

Baew-Christow, Th.; Schwarz, A.; Hör, G. (Frankfurt)

Erfolgreiche immunszintigraphische Tumordetektion mit

Technetium-99m-markierten monoklonalen Anti-CEA-

Antikörpern 26

Pfreundschuh, M.; Da Costa, L.; Diehl, V. (Köln), Carde, P.; Manil, L.; Lumbroso, J.-D.;

Caillou, B.; Boudet, F.; Ricard, M.; Hay at, M.; Parmentier, C. (Villjuif/Frankreich), Saccavini,

J.-C. (Gif-sur-Yvette/Frankreich)

In-vivo-Imaging von Hodgkin-Lymphomen mit monoklo

nalen Antikörpern 30

Gückel, F.; Döhner, H.i Knauf, W.; Ho, A. D.;

Semmler, W.; van Kaick, G. (Heidelberg)

MR-tomographischer Nachweis von Knochenmarkinfil-

trationen bei malignen Lymphomen 34 
Ruhlmann, J.; Bockisch, A.; Dewes, W.; Loos, U.;

Hotze, A.; Biersach, H.-J. (Bonn)

Knochenmarkszintigraphie und Magnetresonanztomo-

graphie beim Plasmozytom 38

Jeske, J.; Herholz,, K.; Heindel, W.; Heiß, W. D. (Köln) Stoffwechseluntersuchungen an

Gliomen mit der Positro-nen-Emissions-Tomographie und der Phosphor-31-MR-Spektroskopie

in Diagnostik und Therapieplanung .... 42

Semmler, W.; Bachert-Baumann, P.; Gückel, F.;

Gademann, G.; Zabel, H.-J.; van Kaick, G. (Heidelberg)

Nicht-invasive Tumortherapieverlaufskontrolle nach

Chemotherapie mit Hilfe der 31P-MR-Spektroskopie an

einem Ganzkörpertomographen

46

Port, R. E.; Strauss, L. G.; Clorius, J. H. (Heidelberg)

Positronen-Emissions-Tomographie nach Kurzinfusion

von 5-[18F]Uracil: Lineares Modell für die Kinetik der

18F-Radioaktivität in Tumoren

51

Supplement 2

ABSTRAKTS zum Symposium der Arbeitsgemeinschaft internistischer Onkologie (AIO)

Chirurgischen Arbeitsgemeinschaft für Onkologie (CAO) Arbeitsgemeinschaft Radiologische

Onkologie (ARO) «Aktuelle Therapie gastrointestinaler Tumoren» 